\title{
Iso-Contraction-Ratio Methodology for the Design of Hypersonic Inward Turning Inlets with Shape Transition
}

\author{
Yabin Xiao $^{1}$, Lianjie Yue. ${ }^{2}$ Lihong Chenr ${ }^{3}$, Xinyu Chang ${ }^{4}$ \\ Institute of Mechanics, Chinese Academy of Sciences, Beijing,100190,China
}

\begin{abstract}
A methodology has been devised for the design of the three-dimensional hypersonic inlets with shape transition. This methodology split the objective three dimensional flowfield into a number of stream tubes, each of which has the contraction ratio that equals to the total contraction ratio of the inlet. Each stream tube is regarded as a portion of an axisymmetric supersonic flowfield designed with computational optimization. All the stream tubes are assembled through a pressure matching process to form the objective three dimensional flowfiled and the inlet surfaces. Computational results show that the inlet has a mass capture of 0.995 at design point of Mach 5.4 and 0.952 at Mach 3.5. The uniformity of flowfield inside the inlet is shown to be quite acceptable.
\end{abstract}

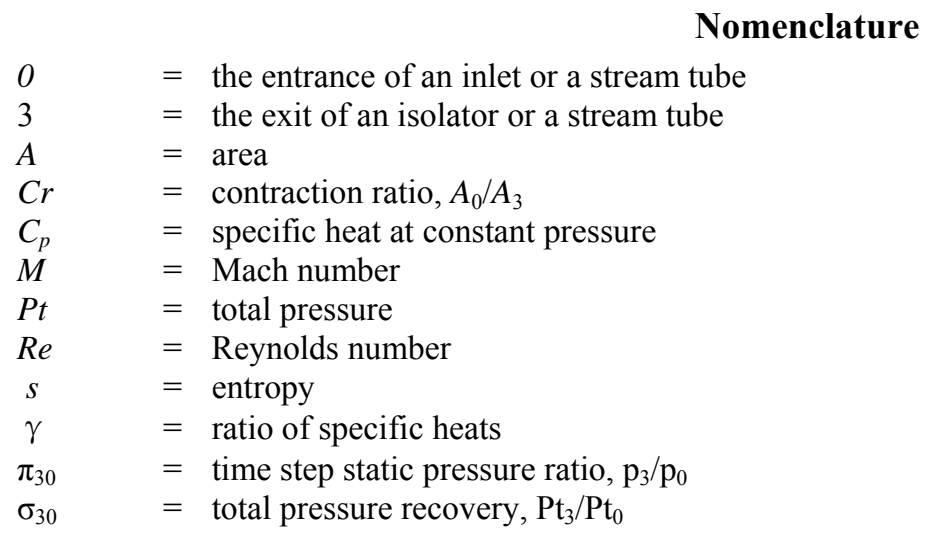

\section{Introduction}

$\mathrm{T}$

HE design methodology for the hypersonic inward turning inlets with shape transitions is a subject of interest in the hypersonic research community. For the modular airframe-integrated scramjets, the capture shape of the hypersonic inlet is preferred to be rectangular like, and at the other hand, a combustor with the circular cross section is superior to a rectangular combustor in terms of the simplicity of the flowfield, the capabilities to withstand the pressure/thermal load and the wetted surface area needed to enclose a specified crosssectional area ${ }^{1}$. Hypersonic inlets designed using three-dimensional shape transition methodologies were believed to satisfy many of these demands. A number of shape transition methodologies were developed in the recent years, which can be classified into three categories, such as (1)the mathematical lofting methods based on the streamline tracing techniques ${ }^{1,2,3},(2)$ the dual-osculating approximation method ${ }^{4,5}$, and (3) the direct computational optimization method ${ }^{7}$.

The REST inlets designed by Smart ${ }^{1}$ is a famous representative that uses the mathematical lofting method based on the streamline tracing techniques. Streamtraced inlets from some known high performance flowfield inherit the

\footnotetext{
${ }^{1}$ Assistant Professor, State Key Laboratory of High Temperature, xiaoyabin@imech.ac.cn.

${ }^{2}$ Associate Professor, State Key Laboratory of High Temperature, yuelj@imech.ac.cn.

${ }^{3}$ Associate Professor, State Key Laboratory of High Temperature

${ }^{4}$ Professor, State Key Laboratory of High Temperature ,AIAA Member. 
favorable features of that. To realize the shape transition, two sets of streamtraced inlet surfaces were derived from the same base flowfield, for one of which, the capture shape of was determined by the rectangular entrance, and for the other the exit shape of was determined by the elliptical throat. Smooth shape transition between the stream traced inlets is accomplished with a mathematical lofting procedure ${ }^{8}$. However, the way in which mathematical lofting affects seriously the inlet performance, and may break some ideal features of the stream traced inlet. Various blend functions were investigated to accomplish the surface morphing ${ }^{2,3}$.

The osculating approximation method developed You ${ }^{4}$ aimed to accomplish an aerodynamic shape transition. The method translated the design problem of a three dimensional flowfield into that of a set of two dimensional conical flowfields. To reduce the pressure gradient between two adjacent slices, the set of conical flowfields were designed to share the same generatrix and have different radial deviation values ${ }^{10}$. However,the reflected shock was then not handled and hence there were additional shock reflection in the isolator.

The direct computational optimization method developed by Sabean ${ }^{7}$ utilized the GASP ${ }^{9}$ CFD code to directly optimize the geometry of a rectangular-to-circular transition. The results showed that the area-averaged standard deviation in pressure across the combustor plane can be reduced to as low as $9.9 \%$ from an initial value of $16.9 \%$ and that the best transition from rectangle to circle for uniform flow is not monotonic. The direct optimization method is believed to be time consuming, especially when the number of optimization variables is increased for better expressing the inlet geometry. The mechanism of the reduction of the standard deviation in pressure still needs to be revealed.

For a scramjet, the compression procedure of inside a hypersonic inlet is essentially a thermodynamic procedure, in which any two independent thermodynamic quantities determines all the other thermodynamic quantities. The uniformity of the flowfield should be expressed in terms of two independent thermodynamic quantities. A flowfield is said to be uniform when and only when two independent thermodynamic quantities of the flowfield are uniform, and this will lead to that all the stream tubes of the flowfield should share the same contraction ratio i.e. the ratio of the entrance area and the exit area.

All the methods mentioned above paid great endeavors to maintain the uniformity of the flowfields, but a necessary condition of which was not dedicated to be satisfied that all the stream tubes in the flowfield should share the same contraction ratio. Based on this idea, an Iso-Contraction-Ratio methodology was developed in this paper for the design of hypersonic inward turning inlet with shape transitions.

\section{One Dimensional Analysis of a Compressible Stream Tube}

Before embarking the design procedure of the inlet, it is important to study the relation between the contraction ratio and the thermodynamic quantities of a compressible stream tube which constitutes the three dimensional flowfield inside the hypersonic inlet. For a compressible stream tube with an entrance area of $\mathrm{A}_{0}$ and an entrance area of $A_{3}$ (shown in Figure 1), the contraction ratio is

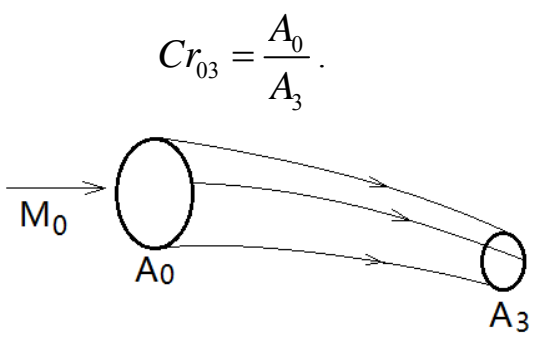

Figure 1. A compressible stream tube.

Without loss of generality, the two thermodynamic quantities are chosen as the quantities usually used to evaluate the performance of a hypersonic inlet, such as the pressure ratio $\pi_{30}$ and the total pressure recovery $\sigma_{30}$. For thermally ideal gases one has the thermal equation of state $\mathrm{p}=\rho \mathrm{RT}$, or the differential form

$$
\frac{d \rho}{\rho}=\frac{d p}{p}-\frac{d T}{T} .
$$

From the Second Law of Thermodynamics, we have the relation

$$
d h=C_{p} d T=\frac{d p}{\rho}+T d s=R T \frac{d p}{p}+T d s,
$$


where $h$ is the specific enthalpy, and hence

$$
\frac{C_{p}}{R T} d T=\frac{d p}{p}+\frac{d s}{R} .
$$

According to (2), the relation of the total temperature $T_{t}$, the total pressure $P_{t}$ and the entropy is

$$
\frac{C_{p}}{R T} d T_{t}=\frac{d P_{t}}{P_{t}}+\frac{d s}{R} .
$$

For a steady flow that the total temperature of the incoming flow remains constant, (3) becomes

$$
\frac{d P_{t}}{P_{t}}+\frac{d s}{R}=0 \text {. }
$$

From (2) and (4) we have

$$
\frac{C_{p}}{R} \frac{d T}{T}=\frac{d p}{p}-\frac{d P_{t}}{P_{t}} .
$$

For a calorically ideal gas, $\gamma$ is a constant, and (5) can be written as

$$
\frac{\gamma}{\gamma-1} \frac{d T}{T}=\frac{d p}{p}-\frac{d P_{t}}{P_{t}}
$$

From the conservation of the mass flow rate $\rho u A=$ const, we have

$$
-\frac{d A}{A}=\frac{d \rho}{\rho}+\frac{d u}{u}
$$

By substituting(1)and (5) into (7), we have

$$
-\frac{d A}{A}=\frac{1}{\gamma} \frac{d p}{p}-\frac{\gamma-1}{\gamma} \frac{d P_{t}}{P_{t}}+\frac{d u}{u}
$$

Integrate (8) along the stream line from 0 to 3 , then we have

$$
C r_{03}=\frac{A_{0}}{A_{3}}=\left(\frac{p_{3}}{p_{0}}\right)^{\frac{1}{\gamma}}\left(\frac{P t_{3}}{P t_{0}}\right)^{-\frac{\gamma-1}{\gamma}} \frac{u_{3}}{u_{0}},
$$

or

$$
C r_{03}=\pi_{30}^{\frac{1}{\gamma}} \sigma_{30}^{-\frac{\gamma-1}{\gamma}} \frac{u_{3}}{u_{0}}
$$

where

$$
\begin{aligned}
& \frac{u_{3}}{u_{0}}=\sqrt{\frac{C_{p}\left(T_{0}-T_{3}\right)+\frac{1}{2} u_{0}^{2}}{\frac{1}{2} u_{0}^{2}}} \\
& =\sqrt{2 C_{p} \frac{1}{\gamma R} \frac{\gamma R T_{0}}{u_{0}^{2}}\left(1-\frac{T_{3}}{T_{0}}\right)+1} \\
& =\sqrt{\frac{2}{(\gamma-1) M_{0}^{2}}-\frac{2}{(\gamma-1) M_{0}^{2}}\left(\frac{\left.\pi_{30}\right)^{\frac{\gamma-1}{\gamma}}}{\sigma_{30}}\right)^{-1}} .
\end{aligned}
$$

By substituting (9) into (8), we have the relation of the contraction ratio $\mathrm{Cr}_{03}$ and the pressure ratio $\pi_{30}$ and the total pressure recovery $\sigma_{30}$ for a compressible stream tube in the inflow Mach number of $\mathrm{M}_{0}$ 


$$
C r_{03}=\pi_{30}^{\frac{1}{\gamma}} \sigma_{30}^{-\frac{\gamma-1}{\gamma}} \cdot \sqrt{\frac{2}{(\gamma-1) M_{0}^{2}}-\frac{2}{(\gamma-1) M_{0}^{2}}\left(\frac{\pi_{30}}{\sigma_{30}}\right)^{\frac{\gamma-1}{\gamma}}+1}
$$

If the flowfield inside an inlet is uniform then any two stream tubes in the flowfield should have the same $\pi_{30}$ and $\sigma_{30}$, and as a result they should share the same contraction ratio. In other words, it is a necessary condition for the uniformity of a compressible flowfield.

According to (10), $\mathrm{Cr}_{03}$ and $\mathrm{M}_{3}$ contour plots with respect to the $\pi_{30}-\sigma_{30}$ plane is shown in Figure 2 when the inflow Mach number is 5.4. $\mathrm{Cr}_{03}$ and $\mathrm{M}_{3}$ contour plots with respect to the $\pi_{30}-\sigma_{30}$ plane is shown in Figure 3 when the inflow Mach number is 3.5 .

For a stream tube with the contraction of $\mathrm{Cr}_{03}, \pi_{30}$ and $\sigma_{30}$ of the stream tube can only change along the contour of $\mathrm{Cr}_{03}$. The contour plots of the $\mathrm{M}_{3}$ are straight lines that pass through the point $(\pi 30, \sigma 30)=(0,0)$. On any $\mathrm{Cr}_{03}$ contour, $\sigma_{30}$ has an minimum value when $\mathrm{M}_{3}=1$. When $\mathrm{M}_{0}$ and $\mathrm{M}_{3}$ are given, the stream tube with a larger $\mathrm{Cr}_{03}$ has higher $\pi_{30}$ and $\sigma_{30}$.

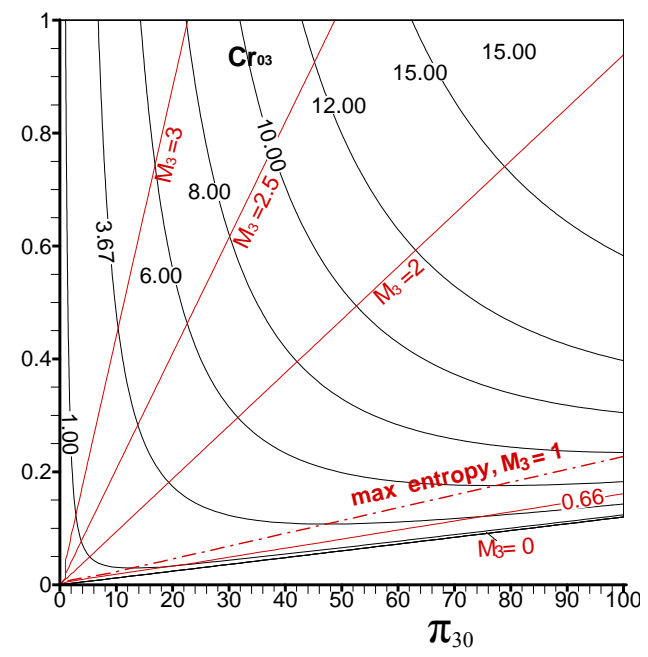

Figure2. Contours of $\mathrm{Cr}_{03}$ with respect to $\pi_{30}-\sigma_{30}$ plane of a stream tube with the incoming Mach number of 5.4.

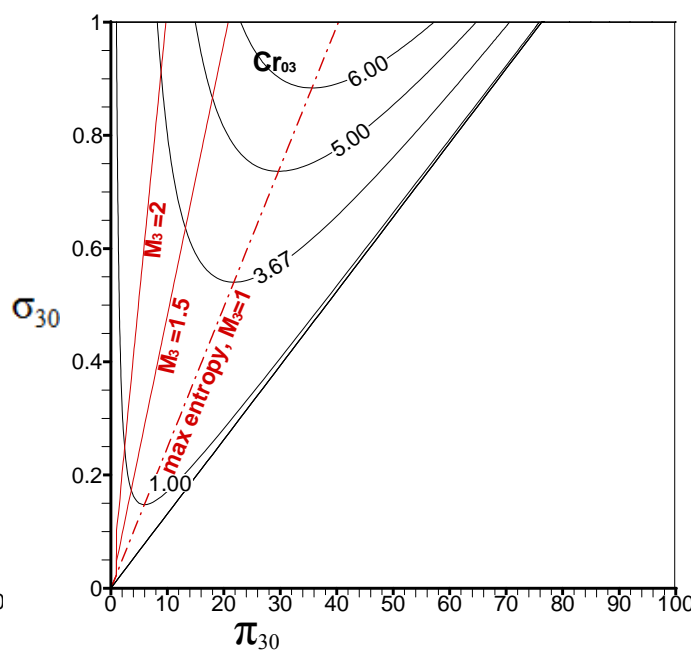

Figure3. Contours of $\mathrm{Cr}_{03}$ with respect to $\pi_{30}-\sigma_{30}$ plane of a a stream tube with the incoming Mach number of 3.5.

For a compression system that incorporates with an initial shock, an isentropic compression and a reflected shock shown in Figure 4, the behavior of the stream tube 0-1-2-3 in the $\pi_{30}$ and $\sigma_{30}$ plane is shown as the black curve in Figure 6, which is named the "compression curve" here. The total pressure recovery $\sigma_{30}$ drops suddenly across the 0-1 and 2-3 procedure, and keeps constant in the 1-2 procedure. Each stream tube has a unique compression curve. If the flowfield of the compression system is uniform then any two independent thermodynamic are uniform, so the compression curve of any other stream tube 0'-1'-2-3' should reach the same end point 3 in Figure 6 . This means any stream tube 0 ' -1 ' $-2-3$ ' and $0-1-2-3$ have the same contraction ratio. This property that any two distinct stream tube share the same contraction ratio is named as "Iso-Contraction-Ratio" in this paper.

However, the Iso-Contraction-Ratio property cannot always be achieved. For a stream tube in the boundary layer, because of the high dissipation, the total pressure recovery $\sigma_{30}$ drops rapidly along the stream line (green curve in Figure 7), and the cross section area grows along the stream lines, and as a result the contraction ratio $\mathrm{Cr}_{03}$ is much less than that of an inviscid stream tube. So the Iso-Contraction-Ratio concept is from the inviscid point of view.

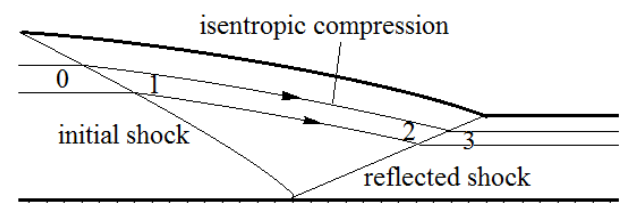

Figure 4. An inviscid stream tube that compressed by a shock-isentropic-shock structure.

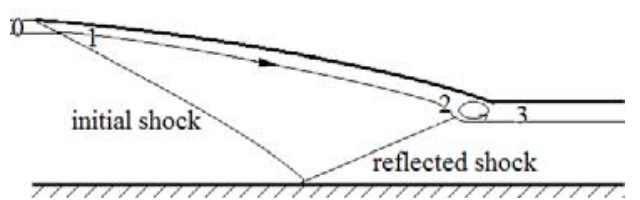

Figure 5. A viscous stream tube in the boundary layer.

4 


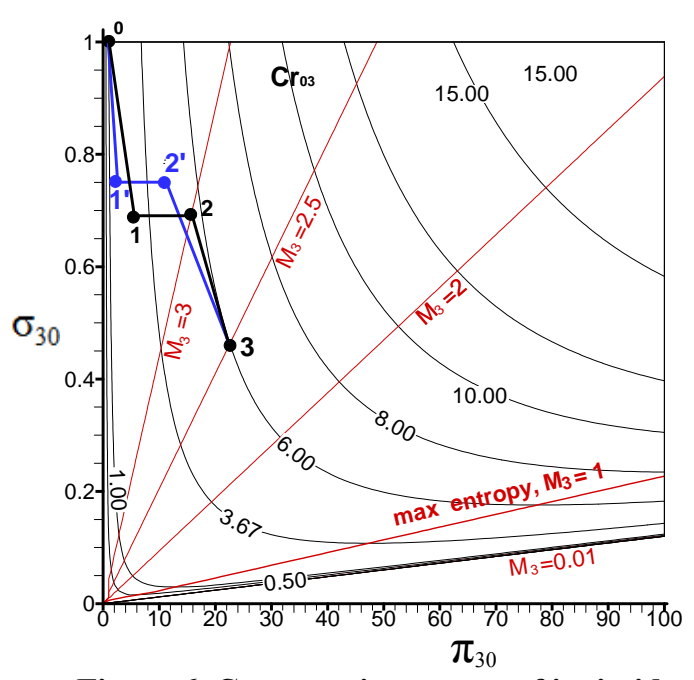

Figure 6. Compression curves of inviscid stream tubes in the $\pi_{30}-\sigma_{30}$ plane.

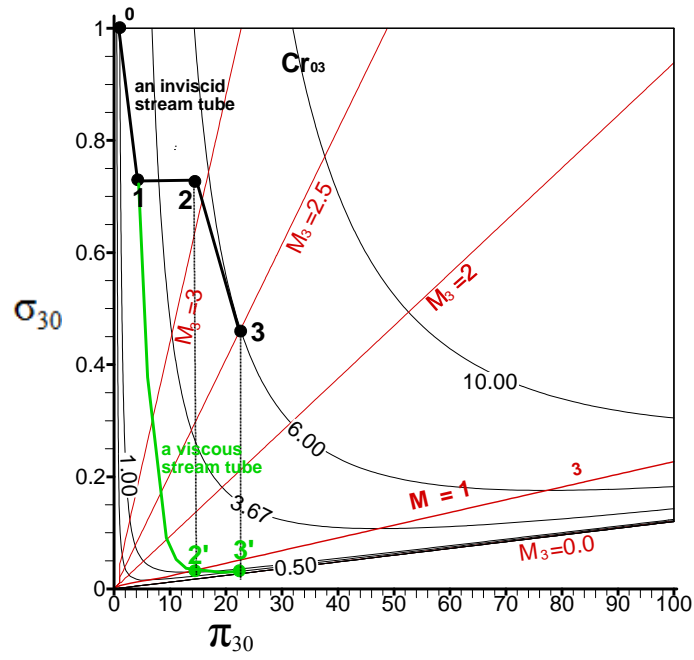

Figure 7. Compression curves of an inviscid and a viscous stream tube in the $\pi_{30}-\sigma_{30}$ plane.

\section{The Iso-Contraction-Ratio Design Methodology}

In order to achieve the Iso-Contraction-ratio property, the objective three dimensional flowfield can be split into a number of stream tubes, each of which has the same contraction ratio as the total contraction ratio of the inlet. Any adjacent two stream tubes (such as ACB-A'C'B' and ABD-A'B'D' in Figure 8) share a common stream surface (ABB'A' in Figure 8) which provides common boundary conditions for the flowfield of each stream tube. All the stream tubes are coupled and need to be designed simultaneously.

As a preliminary research, an approximation was taken in this paper to reduce the coupling of the stream tubes so that each stream tube can be designed individually. A stream tube was regarded as a portion of some base flowfield that can be further designed. All the base flowfields were then designed to have the same contraction ratio and pressure ratio.

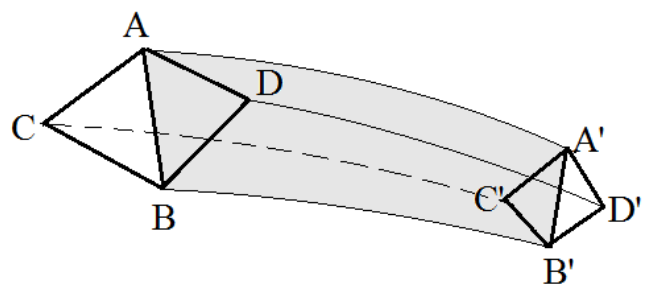

Figure 8. Two adjacent stream tubes sharing a common stream surface.

An inlet with the rectangular capture shape and circular throat shape is shown in Figure 9, the contraction ratio of which is $\mathrm{Cr}_{03} . \mathrm{T}_{0} \mathrm{~W}$ is the symmetric line, and $\mathrm{T}_{0} \mathrm{~W}$ interests the circle at $\mathrm{C}_{0} . \mathrm{T}_{1}$ is a point on the top of the rectangle. For any $T_{1}$, there would be a point $S_{1}$ on $T_{0} W$ satisfying

$$
\frac{A_{\mathrm{S}_{1} T_{1} T_{0}}}{A_{\mathrm{S}_{1} C_{1} C_{0}}}=C r_{03},
$$

where $\mathrm{C}_{0}$ is the intersection point of $\mathrm{T}_{0} \mathrm{~S}_{1}$ and the circle $\mathrm{C}_{0} \mathrm{UW}$ and $\mathrm{C}_{1}$ the is the intersection of $\mathrm{T}_{1} \mathrm{~S}_{1}$ and the circle $\mathrm{C}_{0} \mathrm{UW}$. $A_{\mathrm{S}_{1} \mathrm{~T}_{1} T_{0}}$ is the area of the triangle $\mathrm{S}_{1} \mathrm{~T}_{1} \mathrm{~T}_{0}$. Repeat the above process. For any $\mathrm{T}_{\mathrm{i}}, \mathrm{S}_{\mathrm{i}}$ and $\mathrm{T}_{\mathrm{i}+1}$, there would be a point $\mathrm{S}_{\mathrm{i}+1}$ satisfying 


$$
\frac{A_{S_{i} S_{i+1} T_{i+1} T_{i}}}{A_{S_{i} S_{i+1} C_{i+1} C i}}=C r_{03},
$$

where $C_{i+1}$ is the intersection of $T_{i+1} S_{i+1}$ and the circle $C_{0} U W . T_{i} S_{i}$ intersects $T_{i+1} S_{i+1}$ at $O_{i}$. The front view of flowfield was split in this way into a finite number of blocks.

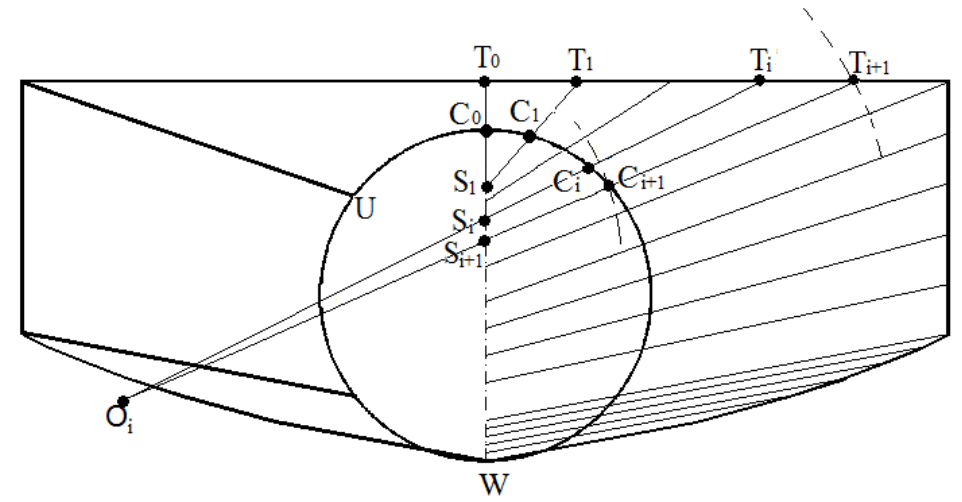

Figure 9. Front view of the split of the flowfield in inlet with rectangular to circular shape transition.

Imagine that there is a hexahedral stream tube with the entrance shape of $S_{i} S_{i+1} T_{i} T_{i+1}$ and with the exit shape of $S_{i} S_{i+1} C_{i+1} C_{i}$. The stream tube is shown in Figure 10, which has the entrance shape of $S_{i} S_{i+1} T_{i} T_{i+1}$ and with the exit shape of $S_{i}{ }^{\prime} S_{i+1}{ }^{\prime} C_{i+1} C_{i}$, where $S_{i}$ 'and $S_{i+1}$ ' are projections of $S_{i}$ and $S_{i+1}$ in the inflow direction. Here it is assumed that the hexahedral stream tube have three planar stream surfaces such as $S_{i+1} S_{i+1} C_{i+1} T_{i+1}, S_{i} S_{i}^{\prime} C_{i} T_{i}$, and $S_{i} S_{i+1}$ $\mathrm{S}_{\mathrm{i}+1}$ ' $\mathrm{S}_{\mathrm{i}}$ ' and one axisymmetric stream surface $T_{i} T_{i+1} C_{i+1} C_{i}$, which is a part of the inlet surface.

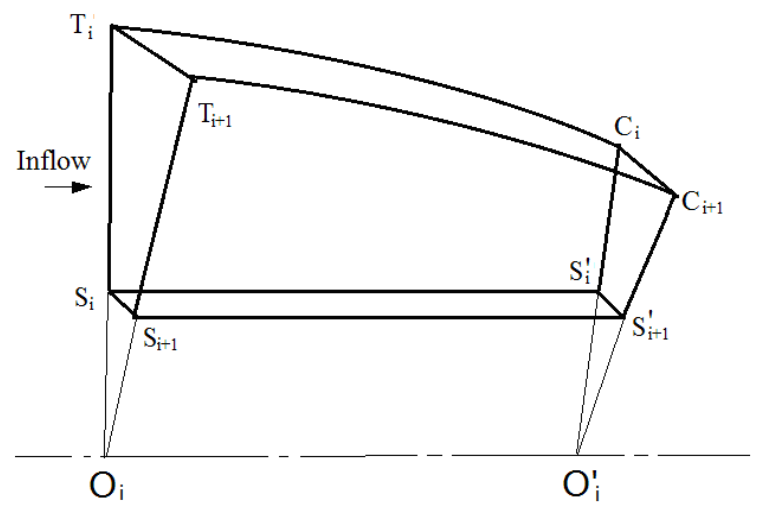

Figure 10. Three-dimensional structure of the hexahedral stream tube that have three planar stream surface.

The stream tube $\mathrm{S}_{\mathrm{i}} \mathrm{S}_{\mathrm{i}+1} \mathrm{~T}_{\mathrm{i}} \mathrm{T}_{\mathrm{i}+1}-\mathrm{S}_{\mathrm{i}}{ }^{\prime} \mathrm{S}_{\mathrm{i}+1}{ }^{\prime} \mathrm{C}_{\mathrm{i}+1} \mathrm{C}_{\mathrm{i}}$ in Figure 10 can be further regarded as an axial portion some axisymmetric flow shown in Figure 11 that can be designed separately. The conical flowfield incorporates with an initial shock, an isentropic compression section and a reflected shock. The initial shock is reflected by the center cylinder with the radius of $\left|\mathrm{O}_{\mathrm{i}} \mathrm{S}_{\mathrm{i}}\right|$ at $\mathrm{P}_{\mathrm{i}}$. The reflected shock intersect the shoulder $\mathrm{B}_{\mathrm{i}}$. Each stream tube belongs to a unique conical flowfield and all the conical flowfields share the same contraction ratio of $\mathrm{Cr}_{03}$. However, as is known to all that for such a conical flowfield the initial conical shock is curved, and the flow downstream the curve initial shock is rotational, so the uniformity of the total pressure recovery cannot be achieved. Fortunately, for the conical flowfield with bigger center cylinder radius, the nonuniformity of the total pressure recovery will reduce significantly.

For each conical flowfield, a NURBS curve with two control points $G_{1}$ and $G_{2}$ was used to express the generatrix. An optimization coupled with the computational flow solver was performed to ensure that all the conical flowfields have the same mass weighed average pressure ratio and that the reflection shock can be canceled in the isolator. The optimization was performed utilizing the General Pattern Search algorithm in the MATLAB optimization toolbox. 
The viscous computations of flowfield were performed utilizing the commercial code FLUENT 2D. The optimization variables were selected to be the vertical coordinates of $G_{1}$ and $G_{2}$.

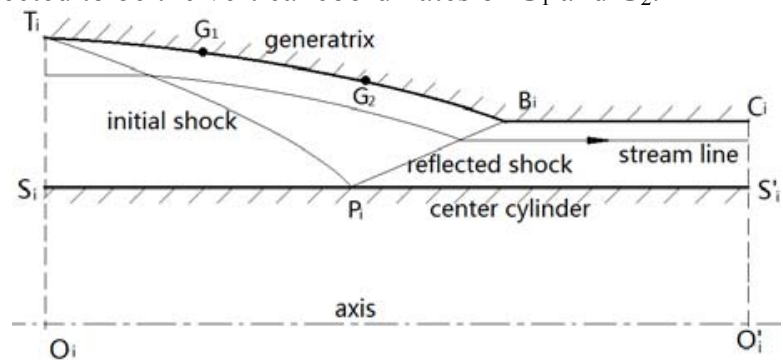

Figure 11. Conical flowfield that contains the

Finally, all the stream tubes as shown in Figure 10 need to be assembled to form the objective flowfield inside the inlet. All the outer stream surface $T_{i} T_{i+1} C_{i+1} C_{i}(i=0, \ldots$,number of the stream tubes) form the surface of the inlet. During the assembly procedure, the shock reflection point $\mathrm{P}_{\mathrm{i}}$ of each stream tube retains the same axial coordinate to reduce the cross flow between any two adjacent stream tubes. The pressure matching process helps to reduce the small cross flow that pass through the designed common boundary of two adjacent stream tube. All the stream tubes were assembled to constitute the objective three dimensional flowfield in this way.

\section{Inlet Geometry}

The hypersonic inlet with the rectangular to circular transition designed using the Iso-Contraction-Ratio methodology is shown in Figure 12. The components of the inlet are illustrated in Figure 13, where 1 represents the leading edge generating the initial shock, 2 the contraction section, 3 the intersection curve of the contraction section and the isolator, i.e. the shoulder, 4 the cylindrical isolator, 5 the circular exit, and 6 the cowl. The inlet has a total length of $1128 \mathrm{~mm}$, the isolator $507 \mathrm{~mm}$, the total width $280 \mathrm{~mm}$ and the total height $115 \mathrm{~mm}$. The design point of the inlet is Mach 5.4.

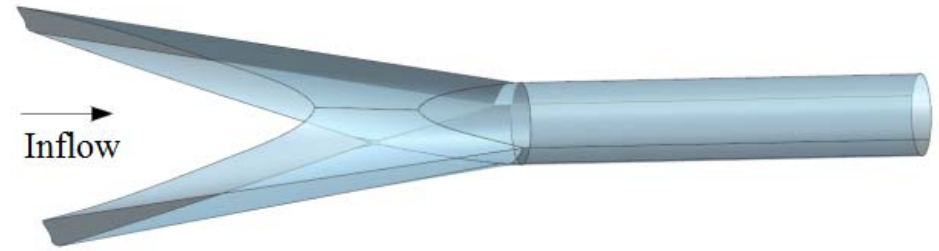

Figure 12. Three-dimensional view of the hypersonic inlet with the rectangular to circular shape transition designed using the Iso-Contraction-Ratio methodology.

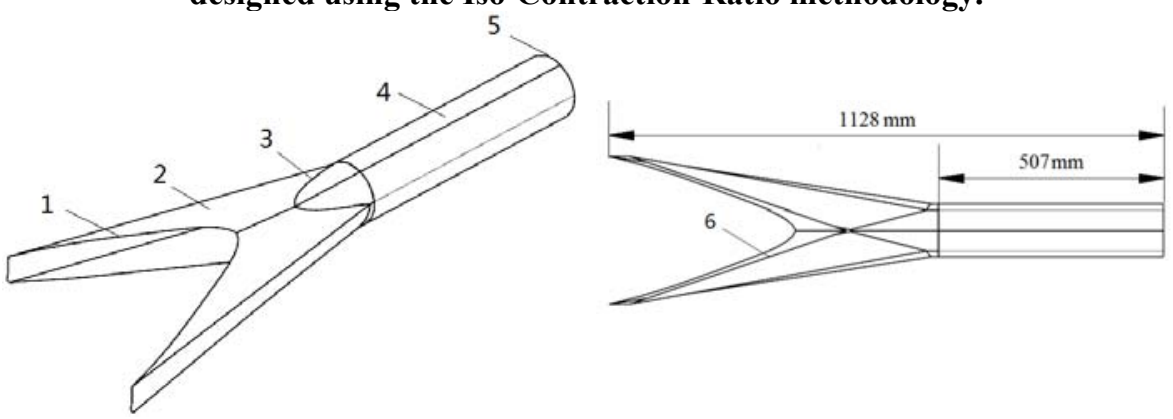




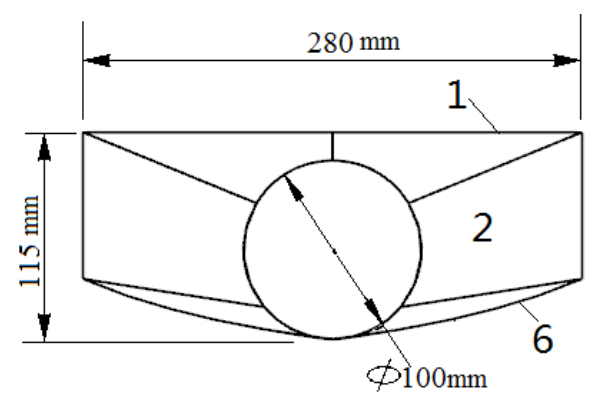

Figure 13. Components and dimensions of the inlet.

\section{Computational Analysis}

The commercial code FLUENT was used to solve the inlet flowfield on typical grids with 1.35 million grid points for the inviscid and 2.09 million rid points for the viscous computation respectively. For the current computations the working fluid was air whose specific heat at constant pressure is a piecewise polynomial of the temperature which is provided by FLUENT. The two-equation $\mathrm{k}-\mathrm{w}$ SST model was used to model the turbulence. The hybrid grid were generated with ICEM-CFD which consisted of the tetrahedral cells in the core flow region and the 28 layers of triangular prism cells to resolve the wall boundary layers. For the viscous grid the height of the first layer is $0.005 \mathrm{~mm}$. Both the external flow below and the internal flow through the inlet were solved. The computations were performed using a parallel version of FLUENT on a HP BL685(4x 2.1 GHz AMD Opteron 6172 12-Core CPU) server. This server runs Ver. 6.0 of the CentOS Linux operating system and uses the MPICH2 form of MPI.

\section{A. Performance of the Inlet}

The inlet in this paper has a design point of the Mach 5.4 and $\operatorname{Re} 4.885 \times 10^{6}$ and a takeover point of Mach 3.5 and $\operatorname{Re} 1.297 \times 10^{7}$. The performance parameters from the viscous computation are tabulated in Table 1 . The inlet mass capture rate at the design point is 0.995 , i.e. the inflow in the capture shape is almost fully captured. The total pressure recovery is 0.52 . The mass capture rate at the takeover point is 0.952 . The inlet suggests a strong mass capture capability.

\begin{tabular}{|l|l|l|l|l|}
\hline Property & $\mathrm{f}_{30}$ & $\pi_{30}$ & $\sigma_{30}$ & $\mathrm{M}_{3}$ \\
\hline Design point & 0.995 & 11.73 & 0.52 & 2.945 \\
\hline Takeover point & 0.952 & 12.26 & 0.684 & 1.578 \\
\hline
\end{tabular}

Table 1. Properties at the exit of the isolator

\section{B. The Structures of the Initial Shock and the Reflected Shock.}

The mass capture ratio at the design point is determined by the shape of the initial shock. If the leading edge and the cowl are wave rider, then the inlet will have the maximum mass capture ratio. Cancellation of the reflected shock is important for weakening the oblique shock train in the isolator.

For better understanding the shape of the initial shock and the reflected shock, an inviscid computation at the design point of Mach 5.4 was performed. As shown in Figure 14, the initial shock that emanates from the top and side leading edges is a non-conical surface. The initial shock is almost ridden by the cowl. The reflected shock is a three-dimensional structure and intersects exactly with the cowl shoulder and closely with the left and right shoulders. However, the reflected shock does not intersect with the top shoulder as designed. The reason for this is that the crosssectional pressure difference is very strong there and this causes strong cross flow between designed stream tubes, and as a result, the Iso-Contraction-Ratio property cannot be retained. Besides initial shock and the reflected shock, there isn't any other shock in the contraction section 


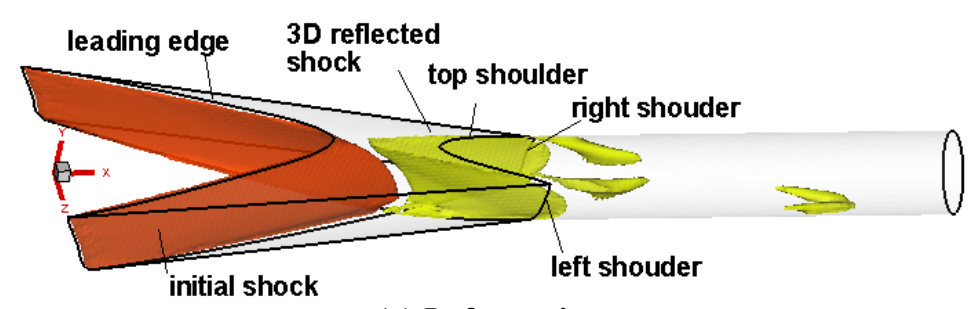

(a) Left-up view

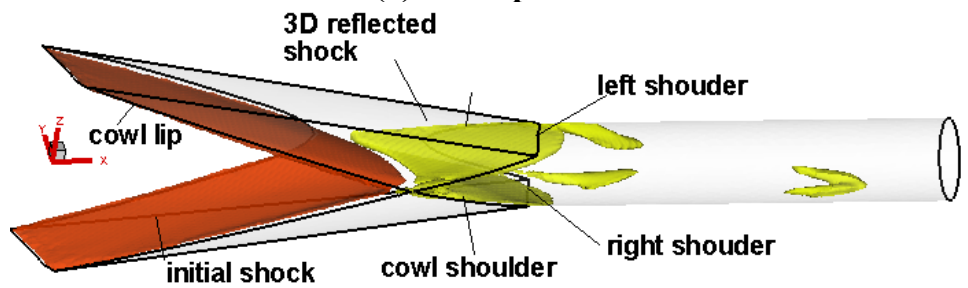

(b) Left-down view

Figure 14. Initial and reflected shock structure of the inlet from an inviscid computation

\section{The viscous flowfield at the design point.}

Eight key slices were created to reveal in detail the viscous flow patterns of the flowfield at the design point which is shown in Figure 15. Slice 1 and 2 were used to check whether the initial shock can be ridden by the cowl lip. Slice 3 was used to check whether the initial shock can be ridden by the crotch of the top leading edge. Slice 4 was used to check whether the initial shock can be ridden by the crotch of the cowl. Slice 5 was used to visualize the structure of the reflected shock. Slice 6,7,8 are the planes at the begining, the middle and the end of the

isolator respectively, and were used to show the uniformity of the flowfield inside the isolator.

As shown in Figure 15, Slice 1 and 2 to show that there is a very small amount of spillage between the initial shock and the cowl. However, the mass capture rate of the inlet was computed to be very closed to 1.0. As shown by Slice 3 and 4, the crotches of top leading edge and that of the cowl are almost riding on the initial shock. From Slice 4 , the "Y" style shock can be observed, which is the intersection of the three dimensional reflected shock and Slice 4. The Mach number distribution between the shock and the wall is uniform in Slice 1,2,3, and 4.

Slice 5 describes the flow patterns inside the internal contraction section, where the "Y" style shock in Slice 4 becomes two separate shocks. Slice 6 describes the flow at the beginning of the isolator, where the Mach number of the core flow ranges from 3.12 to 3.53 . Slice 7 at the middle of isolator, the region where the Mach number is 3.53 has moved to the lower side and the region where the Mach number is 3.12 has moved to upper side, which suggests that there is a horizontal oblique shock in the isolator, but the oblique shock is fairly weak.

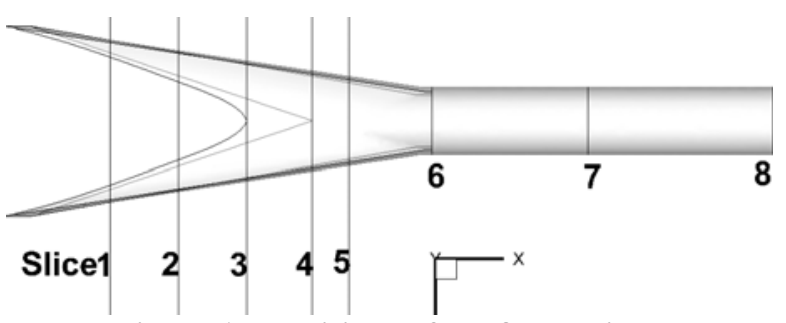

Figure 15. Positions of the 8 key slices. 


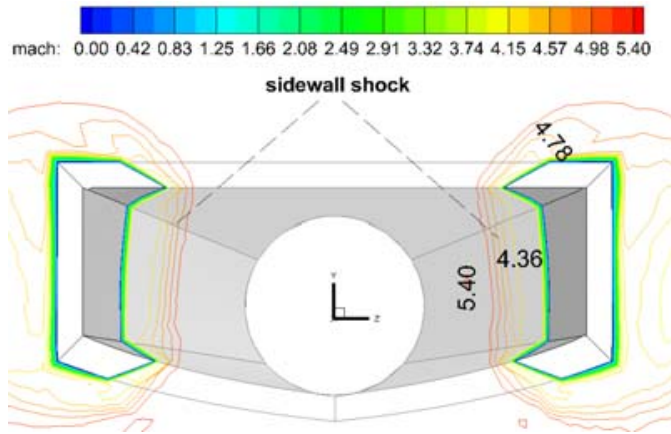

Slice 1

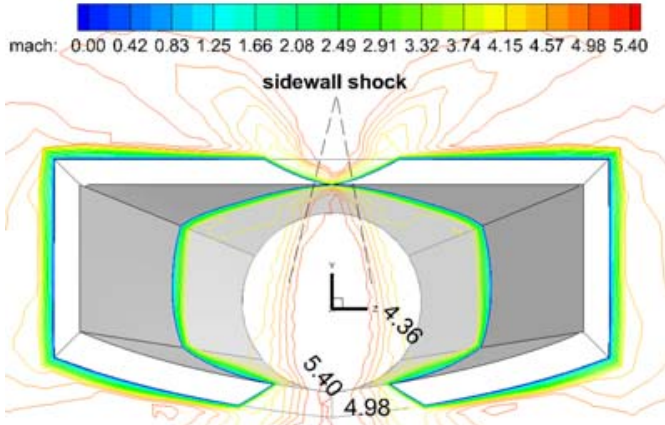

Slice 3

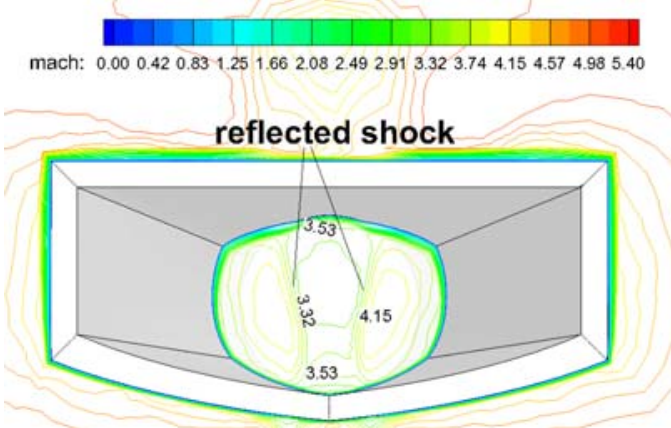

Slice 5

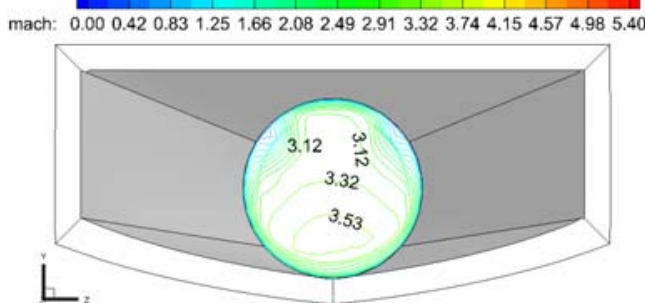

Slice 7

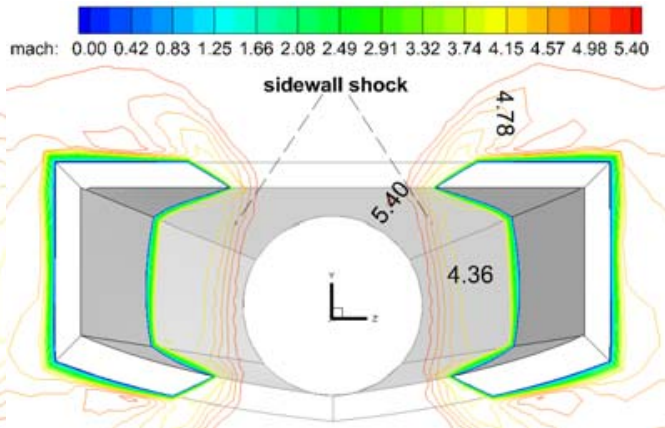

Slice 2

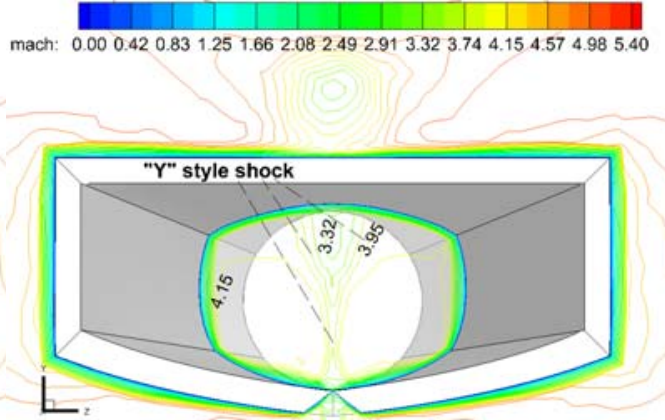

Slice 4

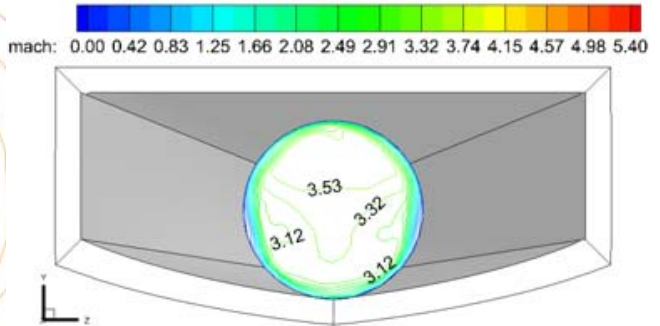

Slice 6

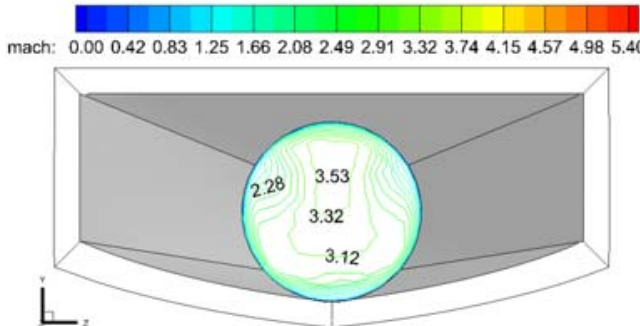

Slice 8

Figure 16. Contours of Mach number in the key slices in Figure 15.

The total pressure recovery slices in $\mathrm{x}$-direction is shown in Figure 17. For clarity, the external flow field is not displayed except in the left most slice. Slice 6 in Figure 17 indicates that the reflected shock didn't cause an obvious boundary layer separation. In a slice inside the isolator, there are two low total pressure regions which are actually two streamwise vortices formed by the upstream boundary layer. Figure 18 is the total pressure recovery distribution. The two-dimensional stream lines are obtained by the y-velocity and the z-velocity in the plane. The spiral point of the stream lines indicate the core of the streamwise vortex. 
Figure 19 is the flowfield in the horizontal slice that passes throught the axis of the cylindrical isolator. The reflected shock was canceled at the inlet shoulder, and the stream lines inside the isolator is quite straight. No strong oblique shock train or boundary layer separation can be observed. The flowfield shows a good horizontal uniformity.

Figure 20 is the flowfield in the symmetry plane of the inlet. There still isn't any boundary layer separation. However, the flowfield uniformity is not as good as that in the above mentioned horizontal plane. The main reason for this the reflected shock doesn't intersect with the top shoulder as designed. The pressure matching of the stream tubes is not as successful at the top of the symmetry plane.

\section{total pressure recovery}

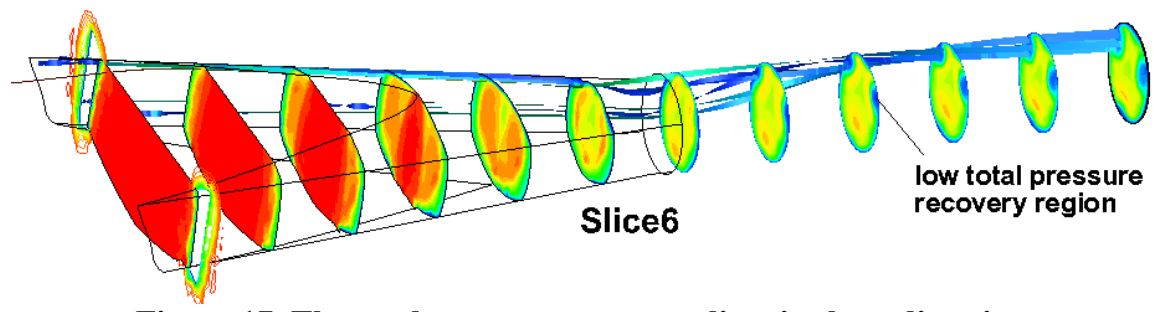

Figure 17. The total pressure recovery slices in the $x$-direction.

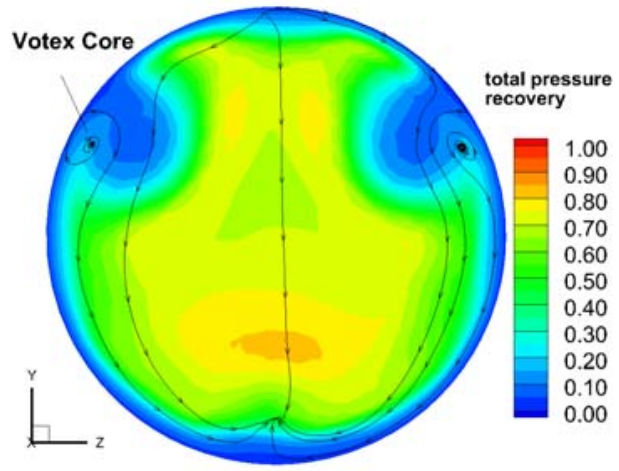

Figure 18. Total pressure recovery distribution at the exit of the Isolator

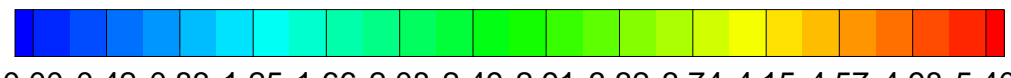

mach: 0.000 .420 .831 .251 .662 .082 .492 .913 .323 .744 .154 .574 .985 .40

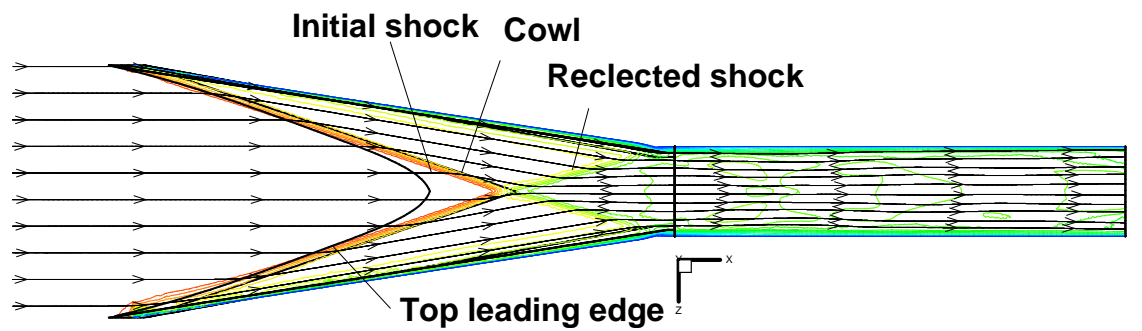

Figure 19. Flowfield in the horizontal slice that passes throught the axis of the cylindrical isolator. 


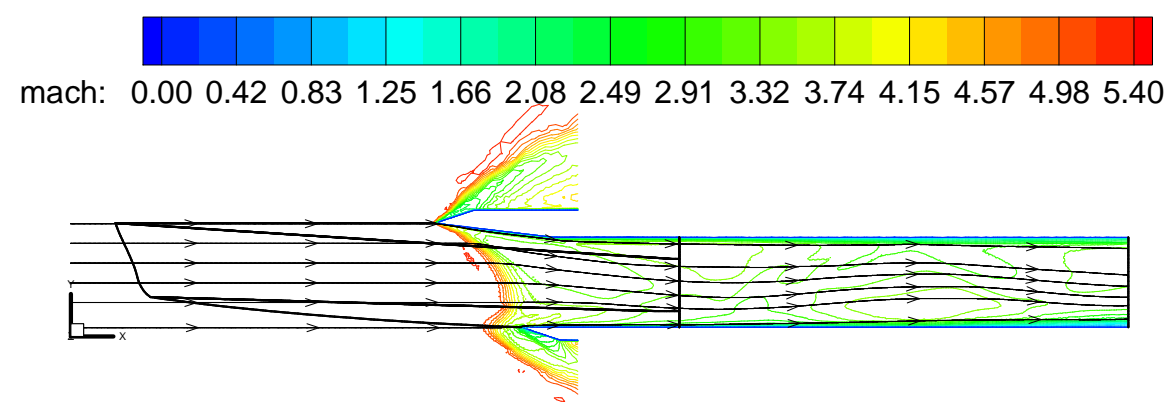

Figure 19. Flowfield in the symmetry plane of the inlet.

\section{Conclusions and Future work}

The relation between the contraction ratio and two independent thermodynamic quantities of a compressible stream tube was studied in this paper. It is revealed that the necessary condition for the uniformity of the flowfield inside an inlet is that all the stream tubes in the flowfield should share the same contraction ratio.

Based on this idea, an Iso-Contraction-Ratio methodology was developed for the design of the hypersonic inward turning inlet. The objective three dimensional flowfield to be designed is split into a number of stream tubes, any two of which shares the same contraction ratio. To reduce the coupling of the stream tubes, an approximation has been taken that small difference between two adjacent stream tube is allowed so that a stream tube can be designed individually. In this paper, a stream tube was assumed to be hexahedral and has three planar stream surfaces and an axisymmetric stream surface. The stream tube was an axial portion of an existing axisymmetric base flowfield. For each stream tube there was a unique axisymmetric base flowfield to be designed. All the base flowfield have the same contraction ratio and pressure ratio.Pressure matching process was taken to reduce small cross flow that pass through the common boundary of two adjacent stream tube. The pressure matching is implemented by matching the intersection point of the initial shock and the reflected shock of the axisymmetric base flowfield.

A hypersonic inward turning inlet with rectangular to circular transition was designed using the Iso-ContractionRatio methodology whose contraction ratio was 3.67. The inlet has a design point of the is Mach 5.4 and Re $4.885 \times 10^{6}$ and a takeover point of Mach 3.5 and Re $1.297 \times 10^{7}$.Computation results show that the inlet has a high mass capture rate as 0.995 at the design point ans 0.952 at the takeover point. The total pressure recovery at the exit of the isolator is 0.520 for the design and and 0.684 for the takeover point respectively.

The initial shock generated by the leading edges is almost ridden by the cowl. Thereflected shock is canceled by the cowl shoulder, the left and the right shoulder, but not canceled by the top shoulder. As a result the flowfield inside the isolator has a pretty good horizontal uniformity and an acceptable but not perfect vertical uniformity. No boundary layer separation occurred in the contraction section. The main reason for this is that the difference between the base axisymmetric flowfield of top most stream tube and that of its neighbor is too great. The Iso-ContractionRatio proper cannot be retained in this region because of the failure of the pressure matching.

The Iso-Contraction-Ratio design methodology is far from mature. In this paper, a stream tube was assumed to be a portion of an axisymmetric supersonic flowfield. For an axisymmetric flowfield with curved initial conical, the flow downstream the initial shock is rotational, so the uniformity of the total pressure recovery cannot be achieved strickly. In fact a stream tube isn't necessary to be a portion of an axisymmetric flowfield. Nevertheless, the boundaries of a stream tube needn't to be planar or axisymmetric. Looking for an appropriate form of a stream tube is a future subject of this work.

The interactions between two adjacent stream tubes were assumed to be small currently. In fact the stream surface as a common boundary of two adjacent stream tubes should really exist. All the stream tubes are coupled through the hyperbolic conservation law. A complete design methodology should be designed and solved simultaneously. The complex algorithm to perform the complete design methodology is a future subject of this work.

\section{References}

${ }^{1}$ M. K. Smart, Design of Three-Dimensional Hypersonic Inlets with Rectangular-to-Elliptical Shape Transition, AIAA Journal of Power and Propulsion, Volume 15, Number 3, pp 408-416, May-June 1999. 
${ }^{2}$ T. Taylor, D. VanWie, Performance Analysis of Hypersonic Shape-Changing Inlets Derived from Morphing Streamline Traced Flowpaths, 15th AIAA International Space Planes and Hypersonic Systems and Technologies Conference, Dayton, Ohio, Apr. 28-1, 2008, AIAA-2008-2635.

${ }^{3}$ XiangJun Nan, Kunyuan Zhang, Zhiguang Jin, Bo Sun, Numerical and Experimental Research of an Inward Turning Inlet with Shape Transition. (in Chinese), Acta Aeronautica et Astronautica Sinica, 2011.7.15, Vol.32, No.6988-996.

${ }^{4}$ Yancheng You, Dewang Liang, Ke Cai,Numerical Research of Three-Dimensional Sections Controllable Internal Waverider Hypersonic Inlet, 4th AIAA/ASME/SAE/ASEE Joint Propulsion Conference \& Exhibit 21 - 23 July 2008, Hartford, CT,AIAA2008-4708.

${ }^{5}$ Sobieczky H, Dougherty F C, Jones K. Hypersonic waverider design from given shock waves. In: 1 St Int Waverider Syrup. CollegePark: American Institute of Aeronautics and Astronautics, 1990

${ }^{6}$ Sobieczky H, Zores B, Zhuo W , et a1. Hi speed flow deisgn using the theory of osculating cones and axisymmetric flows. Acta Aeron Astron Sin, 1999, 12(1): 1- 8

${ }^{7}$ Sabean J W, Lewis M J. Computational Optimization of a Hypersonic Rectangular-to- Circular InletJ. Journal Of Propulsion And Power. 2001, 17(3): 571-578.

${ }^{8}$ Barger, R.L,, "A procedure for designing forebodies with constraints on cross-section shape and axial area distribution", NASA TP 188 1, 198 I.

${ }^{9}$ GASP, Ver. 3.0, Aerosoft, Inc., Blacksburg, VA, 1996.

${ }^{10}$ Kothari A P, Tarpley C, Mclaughlin T A, Hypersonic vehicle design using inward turning flow fields. In: 32nd AIAA / ASME / SAE / ASEE Joint Propulsion Conf Exhibit. Lake Buena Vista: American Institute of Aeronautics and Astronautics, 1996. 